\title{
AVALIAÇÃO DE NANOPARTÍCULAS DE AMIDO COMO ADITIVO A LUBRIFICANTES
}

Matheus Gonçalves Leão de Oliveira1; Pollyana Grazielle Luz da Rocha², Adelson Ribeiro de Almeida Júnior², Jania Betânia Alves da Silva²

1Universidade Federal do Recôncavo da Bahia (UFRB/CETEC); Cruz das Almas/BA; matheusleaoufrb@gmail.com

2Universidade Federal do Recôncavo da Bahia (UFRB/CETEC); Cruz das Almas/BA

Resumo: Este trabalho traz um estudo sobre produção de nanopartículas de amido de mandioca (SNP), por hidrólise ácida, e sua eficiência como aditivo para lubrificantes. O nanolubrificante foi preparado utilizando o óleo Rocol® Ultracut 250, adicionado de SNP's em concentrações variadas $(0,05,0,5,1,00$ e 1,25\% m/m). As nanopartículas foram caracterizadas através da análise do Potencial Zeta e TGA. Para o nanolubrificante (Rocolß Ultracut $250+$ SNP), foi realizado o teste pino-disco, onde foram analisados o coeficiente de atrito $(\mu)$ e a taxa de desgaste $(w)$, antes e depois da adição de SNP. A adição de nanopartículas de amido ao lubrificante apresentou melhorias significativas, como redução do coeficiente de atrito em torno de $30 \%$ e o aumento da taxa de desgaste em mais de $700 \%$.

Palavras-Chave: Nanolubrificante; Nanopartículas de amido; Coeficiente de atrito; Taxa de desgaste

\section{EVALUATION OF STARCH NANOPARTICLES AS A LUBRICANT ADDITIVE}

\begin{abstract}
This research brings a study of cassava starch nanoparticles (SNP) by acid hydrolysis and its efficiency as additive to lubricants. It was conducted with Rocol® Ultracut 250 oil, adding SNP's at varying concentrations $(0.05,0.5,1.00$ and $1.25 \%$ w/w). The SNP's were characterized by Zeta Potential and TGA analysis. For the nanolubricant (Rocol@ Ultracut 250 + SNP), the pin-on-disk test was performed, where the coefficient of friction $(\mu)$ and wear rate $(w)$ were analyzed before and after the addition of SNP. The addition of starch nanoparticles to the lubricant showed significant improvements, such as the reduction of attrition coefficient in $30 \%$ and the increase of wear rate in more than $700 \%$.
\end{abstract}

Keywords: Nanolubricant; Starch nanoparticles; Coefficient of friction; Wear rate 


\section{INTRODUÇÃO}

Atrito, desgaste e lubrificação são parâmetros de estudo da humanidade desde tempos antigos, porém a tribologia foi oficialmente reconhecida como ciência em 1966 pelo governo inglês, com iniciativa de $\mathrm{H}$. Pete Jost [1]. Entender os fenômenos relacionados à interação de superfícies em movimento relativo é à base da tribologia.

Junto à necessidade de compreender e solucionar problemas relacionados às condições tribológica, o avanço da tecnologia e a busca de novos materiais levou a produção de lubrificantes diferenciados, no quesito de aproveitamento energético e vida útil de equipamentos.

Os lubrificantes são materiais utilizados para reduzir os efeitos do atrito e suas consequências (desgaste, aumento de temperatura e etc.). Na usinagem são chamados de fluido de corte, sendo responsável por resfriar e lubrificar a região de contato entre a peça e a ferramenta. [2,3]. No geral, os óleos lubrificantes apresentam aditivos, a fim de obter um fluido adequado às condições de trabalho ao qual o lubrificante é destinado [4]. Quando acrescidos de nanopartículas (metais, óxidos, biopolímeros etc.), recebem o nome de nanolubrificantes. O uso de nanopartículas de $\mathrm{MoS}_{2}$ como aditivo, por exemplo, potencializam a propriedade lubrificante (redução do coeficiente de atrito) além de aumentar a resistência à corrosão e oxidação [5]. Nanotubos de carbono e nanografites, também utilizados como aditivos, aumentam a propriedade lubrificante e também são ótimos condutores térmicos, acarretando propriedades refrigerantes ao fluido [6;7].

O amido é um polissacarídeo formado basicamente por dois polímeros: amilose e amilopectina. A amilose (região amorfa) corresponde cerca de $20 \%$ da estrutura molecular do amido; já a amilopectina (região cristalina), o restante. Suas concentrações variam de acordo com a origem do amido. Em relação ao proveniente da mandioca, a porcentagem de amilose pode variar entre 13,6 e $23,8 \%$. As nanopartículas de amido (SNP) podem ser obtidas através de processos físicos e/ou químicos, como o uso de ultrassom e/ou hidrólise ácida. Quanto a hidrolise, a reação do ácido degrada a região amorfa e encurta as ligações na região cristalina, formando os nanocristais [8; 9].

Provenientes de fontes renováveis e de fácil produção, as nanopartículas de amido (SNP's) apresentam características interessantes para serem aproveitadas como aditivos para lubrificantes, como resistência mecânica e seu potencial biodegradável. Considerando este contexto, este trabalho traz um estudo sobre a produção de nanopartículas de amido de mandioca para utilização como aditivo para lubrificantes.

\section{METODOLOGIA}

\subsection{Síntese Nanopartículas de amido}

A síntese das SNP's foi através de hidrólise ácida seguindo o método proposto por [9], com adaptações. Pesou-se 9,9g de amido de mandioca e dilui-se em $225 \mathrm{~mL}$ de solução de $\mathrm{HCl}$ a $2 \%$. A suspensão foi mantida reservada em tubos Falcon à 
temperatura ambiente durante 49 dias e submetida à agitação diária. Para neutralizar a reação, no $50^{\circ}$ dia, foram feitas 9 (nove) lavagens com água destilada, centrifugando (3800rpm/20min) as amostras, até obter pH neutro.

\subsection{Caracterização das SNP's}

\subsubsection{Potencial Zeta (ろ)}

O potencial zeta $(\zeta)$ [10] é um parâmetro indicador do potencial eletrocinético em um sistema coloidal. A análise foi realizada usando o dispositivo Zetasizer, da série Nano, Malvern Instruments, através de espelhamento de luz. A partir deste, é possível estimar o diâmetro médio das nanopartículas e o $\zeta$.

\subsubsection{Análise Termogravimétrica (TGA)}

A análise termogravimétrica é uma técnica termoanalítica que assiste na perda de massa da amostra em função da temperatura [11]. Para isto, utilizou-se o equipamento Perkin Elmer Pyris 1, com atmosfera inerte $\left(\mathrm{N}_{2}\right)$ com fluxo de $20 \mathrm{~mL} / \mathrm{min}$ e taxa de aquecimento de $20^{\circ} \mathrm{C} / \mathrm{min}$ em uma faixa de temperatura entre 25 e $800^{\circ} \mathrm{C}$.

\subsection{Teste pino-disco}

Para adicionar as SNP's ao Rocol® Ultracut 250 foi necessário diluir o lubrificante, na proporção de 35:1 partes de água destilada e óleo. Foram preparados volumes de $2 \mathrm{~L}$ de nanolubrificantes, cada qual com concentrações de 0,05, 0,5, 1,0 e $1,25 \%(\mathrm{~m} / \mathrm{m})$ de SNP's, em relação à massa do óleo base.

Para o teste, o disco foi acoplado junto a um eixo no torno mecânico e um dispositivo fora montado para acoplar a carga e a esfera. Foram utilizadas arruelas de aço galvanizado com dureza superficial aproximada de 63 HR30T como corpo de prova; e esferas de aço 52100, com dureza aproximada de $800 \mathrm{HV}$ e $5 \mathrm{~mm}$ de diâmetro. Adotou-se uma carga $(0,883 \mathrm{kgf})$ e duas velocidades de rotação (63 e $500 \mathrm{rpm}$ ). Primeiramente realizado a seco - sem adição de lubrificantes; com o Rocol@ Ultracut 250 e assim, com o nanolubrificante (aditivado com nanopartículas de amido).

Torna-se fundamental conhecer precisamente a massa da amostra para análise de desgaste, verificando a massa perdida durante o processo, com ou sem lubrificação. Com este dado, é possível quantificar e analisar a taxa de desgaste, através da equação (1)

$$
\mathrm{W}=\frac{\mathrm{V}}{\mathrm{F} \cdot \mathrm{x}} \frac{\mathrm{mm}^{3}}{\mathrm{Nm}}
$$

Onde w é a taxa de desgaste, $V$ é a perda de volume da amostra, $F(N)$ é a carga aplicada e x é a distância $(\mathrm{m})$ percorrida pelo pino. O volume é correlacionado com a perda de massa e com a massa específica da amostra [12]. Cada teste foi realizado em triplicata. 


\section{RESULTADOS E DISCUSSÕES}

\subsection{Caracterização das SNP's}

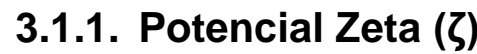

As nanopartículas apresentaram tamanho em uma faixa de 200 e 220nm (Figura 1). Nanopartículas de $\mathrm{MoS}_{2}$ de formato esférico, por exemplo, apresentam maior faixa de distribuição, variando entre 350 a $20 \mathrm{~nm}$, a depender do processo de produção [5]. Nanografites apresentam tamanho em torno de 400-450nm [7].

Figura 1 - Gráfico Potencial Zeta

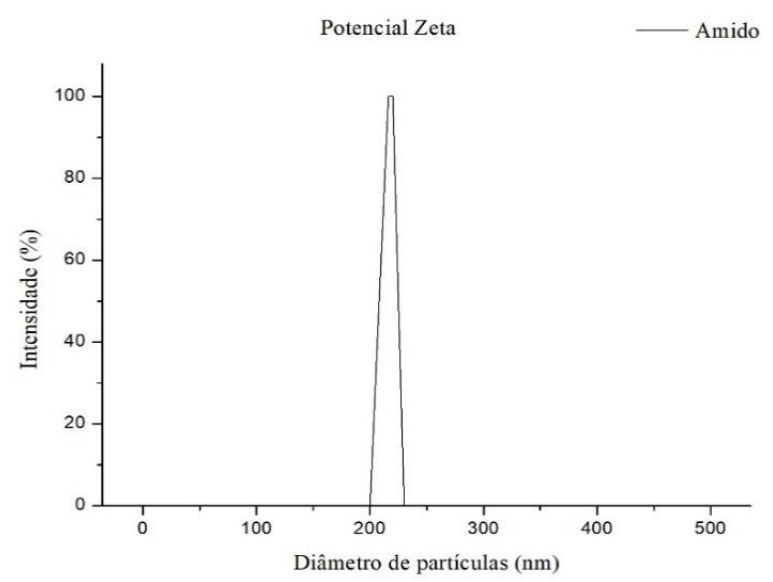

A partir da tabela abaixo (Tabela 1), podemos observar o valor do potencial Zeta $\zeta$, medido em $\mathrm{mV}$

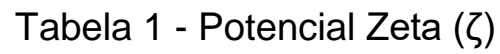

\begin{tabular}{c|c}
\hline № amostra & Potencial Zeta $(\zeta)(\mathbf{m V})$ \\
\hline 1 & $-41,9$ \\
\hline 2 & $-46,8$ \\
\hline 3 & $-45,6$ \\
\hline Resultado $(\bar{\zeta} \pm \Delta \zeta)$ & $(-44,76 \pm 2,55)$ \\
\hline
\end{tabular}

Esta análise indica o valor do potencial de superfície das nanopartículas dispersas no fluido, para conferir a estabilidade da suspensão. É interessante que este valor esteja acima de $30 \mathrm{mV}$ (positivo ou negativo), para que a repulsão eletrostática entre as nanopartículas seja o suficiente para mantê-las dispersas de modo estável [13]. Assim, como o valor do potencial zeta ficou em torno de $-45 \mathrm{mV}$, a dispersão com nanopartículas de amido é considerada estável. 


\section{\begin{tabular}{l|l} 
CIRCULAR ECONOMY & ECONOMIA CIRCULAR
\end{tabular}}

\subsubsection{Análise termogravimétrica (TGA)}

Os resultados obtidos através da TGA estão descritos na Figura 2, onde mostra a curva característica da análise termogravimétrica, correlacionando a perda de massa da amostra (expressa em \% de massa residual) em função da temperatura.

Figura 2 - Curva TGA das nanopartículas de amido.

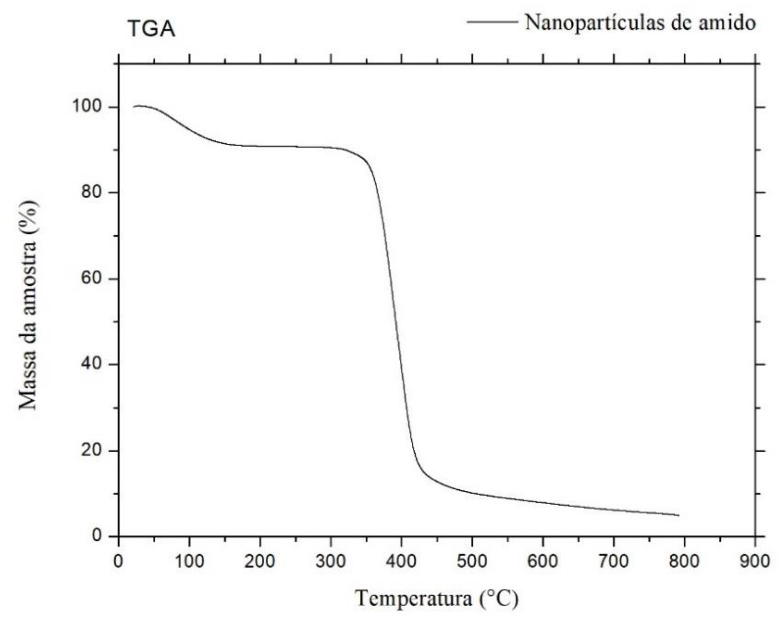

Pela curva de TG, observam-se dois eventos de perda de massa. O primeiro evento pode ser associado à perda de umidade da amostra, ocorrendo até $110^{\circ} \mathrm{C}$. $\mathrm{O}$ segundo evento corresponde à degradação do amido, iniciando a perda de massa em aproximadamente em $340^{\circ} \mathrm{C}$. Assim, pode-se inferir que as SNP's apresentam estabilidade térmica em aproximadamente $340^{\circ} \mathrm{C}$. Nanolubrificantes a base de nanocompósitos de polipropileno/grafite possuem resistência térmica em torno de 300 à $440^{\circ} \mathrm{C}$ [14]. Os nanotubos de carbono apresentam estabilidade térmica em aproximadamente $400^{\circ} \mathrm{C}$ [15]. Mesmo apresentando estabilidade térmica um pouco inferior às nanopartículas utilizadas mais comumente como nanolubrificantes, as SNP's mostram potencial térmico para serem estudadas como aditivo a lubrificantes.

\subsection{Teste pino-disco}

Um dinamômetro acoplado no sistema montado para a execução do teste pinodisco permitiu mensurar a intensidade da força de atrito do processo, e determinar o coeficiente de atrito $(\mu)$. Na tabela 2 estão expressos os valores médios de perda de massa com o nanolubrificante (Rocol® Ultracut $250+$ SNP)

Tabela 2 - Massa removida de cada teste pino-disco com os nanolubrificantes

\begin{tabular}{c|c|c}
\hline & Teste pino-disco| Tempo: 15min | Carga: 8,66N \\
\hline $\begin{array}{c}\text { Rotação } \\
\text { (rpm) }\end{array}$ & Especificação do teste & $\begin{array}{c}\text { Resultado } \\
(\bar{m} \pm \Delta \mathrm{m})(\mathbf{m g})\end{array}$ \\
\hline & Rocol@ Ultracut 250 & $(0,40 \pm 0,20)$ \\
\hline & Roco|® Ultracut $250+\mathrm{SNP}_{0,05 \% .}$ & $(0,20 \pm 0,10)$ \\
\hline
\end{tabular}


\begin{tabular}{l|l} 
CIRCULAR ECONOMY & ECONOMIA CIRCULAR
\end{tabular}

\begin{tabular}{c|c|c}
\hline 63 & Rocol@ Ultracut $250+\mathrm{SNP}_{0,50 \% .}$ & $(0,80 \pm 0,60)$ \\
\hline & Rocol@ Ultracut $250+\mathrm{SNP}_{1,00 \% .}$ & $(0,90 \pm 0,60)$ \\
\hline & Rocol@ Ultracut $250+\mathrm{SNP}_{1,25 \% .}$ & $(0,30 \pm 0,17)$ \\
\hline
\end{tabular}

\begin{tabular}{|c|c|c|}
\hline & Rocol® Ultracut 250 & $(0,40 \pm 0,30)$ \\
\hline & Rocol® Ultracut $250+\mathrm{SNP}_{0,05 \%}$ & $(4,20 \pm 2,60)$ \\
\hline \multirow[t]{3}{*}{500} & Rocol® Ultracut $250+\mathrm{SNP}_{0,50 \%}$ & $(1,40 \pm 0,20)$ \\
\hline & Rocol® Ultracut $250+\mathrm{SNP}_{1,00 \%}$ & $(1,00 \pm 0,40)$ \\
\hline & Rocol® Ultracut $250+$ SNP $_{1,25 \%}$. & $(1,60 \pm 0,15)$ \\
\hline
\end{tabular}

A adição de SNP's ao lubrificante aumentou a remoção de massa, nas duas rotações. Para o teste a 63rpm, é possível observar que houve um aumento gradual na massa removida entre as concentrações de 0,05 e 1,00\% de SNP's; porém, para $1,25 \%$ não houve alteração da massa removida, se comparadas ao teste com Rocol@ Ultracut 250 puro. No teste com rotação de 500rpm, observa-se que a adição de 0,05\% de SNP's causou um aumento abrupto da remoção de massa e, logo depois, um decréscimo para as concentrações seguintes; exceto, para 1,25\% (Figura 3a). Talvez a quantidade de SNP's referente a $0,05 \%$ foi o suficiente para provocar interações físico-químicas capazes de retirar maior material que as demais.

Figura 3 - Massa removida (a) e taxa de desgaste (w) (b) em função da concentração de SNP's.
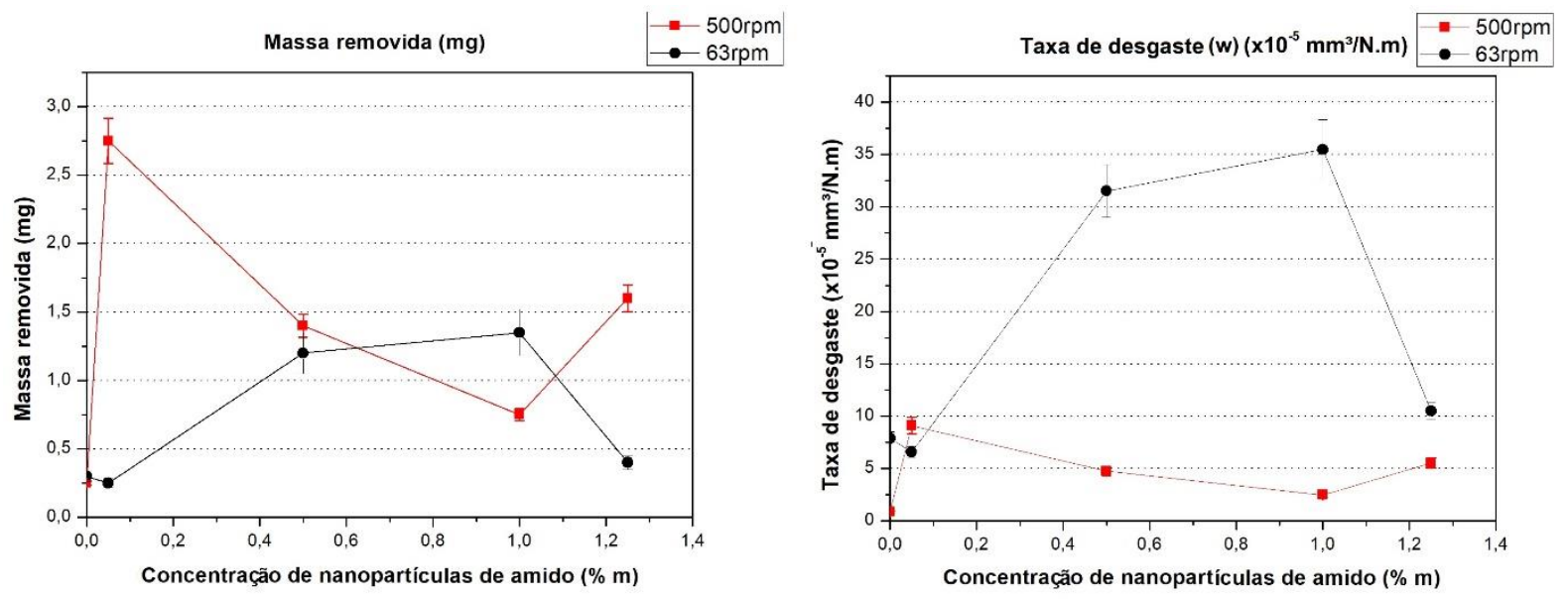

A taxa de desgaste (Figura 3b) na rotação de 63rpm apresenta maiores valores. Como a taxa de desgaste também depende da distância percorrida (ver Equação 1), justifica que para essa rotação apresente maiores valores mesmo com menor massa removida, se comparada aos testes a 500rpm. Interessante observar a taxa de desgaste aumentou em cerca de $400 \%$ para as concentrações de 0,50 e 1,00\% de SNP's. Quando observamos os testes a 500rpm, vemos que a taxa de desgaste aumentou em mais de $1000 \%$ com a adição de 0,05\% de SNP's; para a concentração de $1,25 \%$, a taxa aumentou em cerca de $665 \%$. Com base nesses resultados, podese inferir que as nanopartículas de amido têm caráter abrasivo, pois ajudam na remoção de material da peça, em determinadas concentrações. 
Na figura 4 percebe-se que, para os testes a 63rpm, houve redução significativa do coeficiente de atrito com adição de $0,05 \%$ de SNP's (cerca de $26 \%$ ) e, para o teste a 500rpm, vemos que houve redução de $\mu$ em cerca de $35 \%$ com adição de $1,25 \%$ de SNP's. Analisando os três parâmetros (remoção de massa, taxa de desgaste e coeficiente de atrito), observa-se que para a concentração de 1,25\% de SNP's, temos um fluido de corte com ação abrasiva e lubrificante, pois além aumentar a taxa de desgaste, promove a redução do coeficiente de atrito. Portanto a concentração de nanopartículas no lubrificante estudado influência de forma diferente em cada parâmetro estudado.

Figura 4 - Gráfico do coeficiente de atrito $(\mu)$ em relação a concentração de SNP's, para as duas rotações.

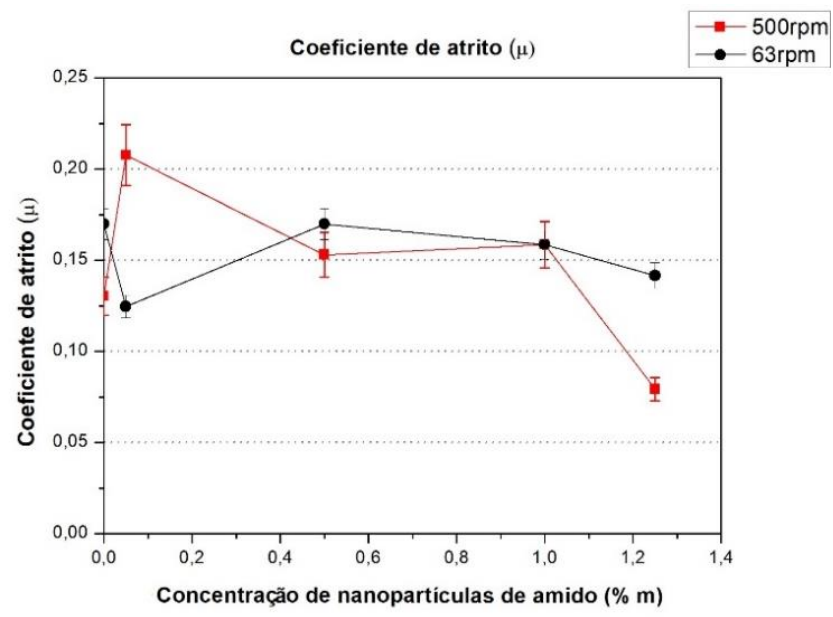

\section{CONCLUSÃO}

A síntese de nanopartículas de amido através da hidrólise ácida foi eficiente, assim como sua adição ao lubrificante Rocol® Ultracut 250. Segundo a análise dos resultados do teste tribológico, pode-se inferir que as SNP's têm naturalmente características abrasivas (aumento de w em até $1100 \%$ ), mas podendo se comportar como agente deslizante (redução de $\boldsymbol{\mu}$ em até $35 \%$ ). Como se trata de nanopartículas, se faz necessário mais testes para entender seu comportamento.

\section{REFERÊNCIAS}

${ }^{1}$ RADI, P. A.; SANTOS, L. V.; BONETTI, L. F.; TRAVA-AIROLDI, V. J. Tribologia, Conceitos e Aplicações. Anais do $13^{\circ}$ Encontro de Iniciação Científica e PósGraduação do ITA - XIII ENCITA / 2007, São José dos Campos, São Paulo, Brasil, ano 2007, 2007.

${ }^{2}$ MAIA, D. A. S. et. al. Fluidos de corte: novas formulações e recuperabilidade. In: Congresso Brasileiro de Pesquisa e Desenvolvimento em Petróleo e Gás, 4., 2007, Campina: ABPG, 2007. p. 1-10.

3JULIÃO, J. C. Estudo comparativo de óleos lubrificantes básicos minerais. Revista da Graduação, Pontifícia Universidade Católica do Rio Grande do Sul. Porto Alegre, v. 4, n. 2, p. 5-21, dez. 2011. 
${ }^{4}$ NETO, W. B. Parâmetros de qualidade de lubrificantes e óleo de oliva através de espectroscopia vibracional, calibração multivariada e seleção de variáveis. 2005. 130 p. Tese (Doutorado em Química) - Instituto de Química, Universidade Estadual de Campinas, Campinas, 2005.

${ }^{5}$ BERNARDI, C. Produção e caracterização de nanopartículas de MoS2 e sua estabilização em óleos visando a lubrificação limite. 2011. 130 p. Tese (Doutorado em Ciências e Engenharia de Materiais) - Departamento de Engenharia Mecânica, Universidade Federal de Santa Catarina, Florianópolis, 2011

${ }^{6}$ CHEN, C. S.; CHEN, X. H.; XU, L. S.; YANG, Z.; LI, W. H. Modification of multi-walled carbon nanotubes with fatty acid and their tribological properties as lubricant additive. Carbon, Elsevier, PR China, v. 43, n. 8, p. 1660-1666, 13 mar. 2005.

${ }^{7}$ OLIVEIRA, A. M. et al. Nanolubrificantes: caracterização de óleo lubrificante modificado com nanografite. Tecnologia e Inovação, Revista IPT, São Paulo, v. 1, n. 2, p. 37-47, ago. 2016.

${ }^{8}$ DENARDIN, C. C.; SILVA, L. P. Estrutura dos grânulos de amido e sua relação com propriedades físico-químicas. Ciência Rural, Santa Maria, p. 1-10, out. 2008.

${ }^{9}$ GONÇALVES, P. M. Obtenção de nanoamido de pinhão através de hidrólise ácida e ultrassom para incorporação da Nisina. 2013. 103 f. Dissertação (Mestrado em Ciência e Tecnologia de Alimentos) - Instituto de Ciência e Tecnologia de Alimentos, Universidade Federal do Rio Grande do Sul, Porto Alegre, 2013.

${ }^{10}$ SOUSA, E. H. V. Obtenção e caracterização de nanolubrificantes utilizados em refrigeração aditivados com nanopartículas de Índio (In). 2017. 198 f. Tese (Doutorado em Engenharia Mecânica) - Centro de Tecnologia, Universidade Federal Rio Grande do Norte, Natal, 2017.

${ }^{11}$ DENARI, G. B.; CAVALHEIRO, E. T. G. Princípios e Aplicações de Análise Térmica. 2012. 40 p. Material de Apoio (Curso Teórico/Prático) - Instituto de Química de São Carlos, Universidade de São Paulo, São Carlos, 2012

${ }^{12}$ CASTRO, R. et al. Desenvolvimento do sistema de aquisição e supervisão de dados para um tribômetro rotativo do tipo pino sobre disco. Revista Ciência e Tecnologia, [S.I.], v. 18, n. 32, p. 1-8, jan. 2015.

${ }^{13}$ SANTOS, C. C. Síntese de nanopartículas de sulfeto de cobre, preparação e estabilidade coloidal de fluidos trocadores de calor em meio aquoso. 2015. $85 \mathrm{f}$. Dissertação (mestrado) - Universidade Estadual Paulista Júlio de Mesquita Filho, Instituto de Química, 2015.

${ }^{14}$ KALINKE, A. H.; ZARBIN, A. J. G. Nanocompósitos entre nanotubos de carbono e nanopartículas de platina: preparação, caracterização e aplicação em eletro-oxidação de álcoois. Quim. Nova, [S.I.], v. 36, n. 8, p. 1289-1296, jun. 2014.

${ }^{15}$ MONTAGNA, L. S. Síntese de nanocompósitos de polipropileno/grafite obtidos por meio da polimerização in situ. 2010.117 p. Dissertação (Mestrado em Engenharia e Tecnologia de Materiais) - Faculdade de Engenharia, Pontifícia Universidade Católica do Rio Grande do Sul, Porto Alegre, 2010. 